\title{
Surface Lattice Resonances in Self-Assembled
}

\section{Gold Nanoparticle Arrays: Impact of Lattice}

\section{Period, Structural Disorder, and Refractive Index}

\section{on Resonance Quality}

Ekaterina Ponomareva, ${ }^{\dagger}$ Kirsten Volk, ${ }^{\dagger}$ Paul Mulvaney, ${ }^{\ddagger}$ and Matthias Karg*, ${ }^{*}$

$\dagger$ Institut für Physikalische Chemie I: Kolloide und Nanooptik, Heinrich-Heine-Universität Düsseldorf, Universitätsstraße 1, D-40225 Düsseldorf, Germany

$\ddagger A R C$ Centre of Excellence in Exciton Science, School of Chemistry, The University of Melbourne, Parkville, VIC 3010, Australia

E-mail: karg@hhu.de

\section{Supporting Information}

\section{Contents}

List of Figures . . . . . . . . . . . . . . . . . . . . . S2

Synthesis of Gold Nanoparticle Cores . . . . . . . . . . . . . . . . . . . S S3

Synthesis of Au-PNIPAM Core-shell Particles . . . . . . . . . . . . . . S3

Overgrowth of Plasmonic Cores . . . . . . . . . . . . . . . . . S4

Characterization of the Au-PNIPAM Core-shell Particles . . . . . . . . . . . S4

Microstructure Analysis of Particle Monolayers _. . . . . . . . . . . . . S6 
Experimental Spectra of all Particle Monolayers . . . . . . . . . . . . . . S10

Optical Response of an Unordered Particle Monolayer . . . . . . . . . . . . . . S10

Local Transmission Spectra of Particle Monolayers . . . . . . . . . . . . . S11

Simulation Layout for FDTD . . . . . . . . . . . . . . . . . . . S12

Theoretical Q-factor for Various Domain Sizes . . . . . . . . . . . . . . S13

Theoretical Optical Response in the Strongest Coupling Regime . . . . . . . . . . S14

References . . . . . . . . . . . . . . . . . . . S15

\section{List of Figures}

S1 TEM Images and UV-Vis Spectra of the Particles . . . . . . . . . . . S5

S2 Microstructure Analysis by DFM . . . . . . . . . . . . S6

S3 Microstructure Analysis by AFM . . . . . . . . . . . . . S7

S4 Analysis of Domain Sizes . . . . . . . . . . . . . . . . . . . S9

S5 Experimental Absorbance Spectra for all Particle Monolayers . . . . . . . . . S10

S6 Optical Response of an Unordered, Dilute Particle Monolayer . . . . . . . . S11

S7 Local Optical Response . . . . . . . . . . . . . . . . . . . . . . . . . S12

S8 Schematic Representation of the Experimental Transmission Measurements and the FDTD Simulation Conditions for the Infinite Lattice . . . . . . . S13

S9 Comparison of Q-factors Obtained from FDTD Simulations . . . . . . . . . S14

S10 Optical Response in the Strong Coupling Regime . . . . . . . . . . . . . S15 


\section{Synthesis of Gold Nanoparticle Cores}

Spherical gold nanoparticles were synthesized according to the protocol of Turkevich et al. ${ }^{1}$ In brief, $200 \mathrm{~mL}$ aqueous gold(III)-chloride trihydrate solution $\left(5 \times 10^{-4} \mathrm{M}\right)$ were brought to boil while stirring. Then $10 \mathrm{~mL}$ of an aqueous sodium citrate dihydrate solution $(1.0 \mathrm{wt} \%$, hot) were added as reducing agent. The dark red nanoparticle dispersion was allowed to boil for another 20 minutes under continuous stirring. After that, the dispersion was allowed to cool down to room temperature under slow stirring. $1.2 \mathrm{~mL}$ of an aqueous SDS solution (1 $\mathrm{mM}$ ) were added to increase the stability of the gold nanoparticles during the purification steps later on. After another 20 minutes, the surface of the particles was functionalized by adding $0.652 \mathrm{~mL}$ of an aqueous butenylamine hydrochloride (BA) solution $(1.4 \mathrm{M}) .^{2}$ The dispersion was left to equilibrate for another 20 minutes. Afterwards the nanoparticles were purified and concentrated via centrifugation for 14 hours at $1400 \mathrm{rcf}$.

\section{Synthesis of Au-PNIPAM Core-shell Particles}

The encapsulations of the gold cores were achieved by seeded precipitation polymerizations according to the protocol by Rauh et al. ${ }^{3}$ The polymerizations were carried out in $100 \mathrm{~mL}$ of Milli-Q water in three-neck round-bottom flasks $(250 \mathrm{~mL})$ equipped with a reflux condenser. For each synthesis NIPAM and BIS were dissolved in water while stirring at room temperature. The respective amounts of NIPAM and BIS are listed in Table 1 in the main manuscript. The solutions were heated to $70{ }^{\circ} \mathrm{C}$ and degassed with nitrogen. After an equilibration time of 20 minutes, $7.37 \mathrm{~mL}$ of the functionalized gold nanoparticles in water $\left(\mathrm{c}\left(A u^{0}\right)\right.$ $=3.137 \mathrm{mM}$ ) were added dropwise. Following another equilibration time of 15 minutes, 2 mg PPS dissolved in $1 \mathrm{~mL}$ of water were added quickly to the mixtures. The polymerization reactions were allowed to proceed for two hours and then cooled down to room temperature. The Au-PNIPAM particles were purified by centrifugation for 90 minutes at $8400 \mathrm{rcf}$ and redispersion of the residues in water. The purification was repeated three times. After the last centrifugation step, the core-shell particles were redispersed in $5 \mathrm{~mL}$ of Milli-Q water. 


\section{Overgrowth of Plasmonic Cores}

The overgrowth protocol used to increase the gold core size was adapted from Honold et al. ${ }^{4}$ Ascorbic acid and $\mathrm{HAuCl}_{4}$ were used in a molar ratio of 1.3. First $8 \mathrm{~mL}$ of an aqueous CTAC solution $(2.4 \mathrm{mM})$ were filled in an Erlenmeyer flask. $93 \mu \mathrm{L}$ of an aqueous solution of the respective Au-PNIPAM particles $(5.04 \mathrm{wt} \%)$ were mixed in a 1:1 ratio with $10 \mathrm{mM}$ CTAC solution and added to the flask under continuous stirring. Then $156 \mu \mathrm{L}$ of an aqueous ascorbic acid solution $(49 \mathrm{mM})$ were added quickly. Next $12 \mathrm{~mL}$ of an aqueous solution containing CTAC $(6.2 \mathrm{mM})$ and $\mathrm{HAuCl}_{4}(0.5 \mathrm{mM})$ were added dropwise under strong stirring. The reactions were allowed to proceed for 30 minutes. The overgrowth steps were repeated four times while the particle size was controlled via extinction spectroscopy aiming at $\lambda_{L S P R}=580 \mathrm{~nm}$, which corresponds to a gold core diameter of approximately 100 $\mathrm{nm}$. The reaction solutions were allowed to rest overnight without stirring. Afterwards the dispersions were purified by three iterations of centrifugation at $5200 \mathrm{rcf}$ for 30 minutes and redispersion of the respective residues in Milli-Q water.

\section{Characterization of the Au-PNIPAM Core-shell Particles}

Figures S1 (a)-(c) show representative TEM images of the initial gold cores from the Turkevich synthesis (a), the Au-PNIPAM particles after seeded precipitation polymerization (b) and the final core-shell particles Au-PNIPAM 367 after overgrowth of the cores (c). Before overgrowth, the cores have an average diameter of $d_{\text {core }}=15.3 \pm 1.3 \mathrm{~nm}$. After the overgrowth, the average core diameter is $d_{\text {core }}=96 \pm 9 \mathrm{~nm}$. The core-shell structure in (b) and

(c) is clearly visible due to the good electron contrast between core and shell. Figure S1 (d) shows absorbance spectra for each type of particle. The gold cores prior to encapsulation into PNIPAM feature a single LSPR at $\lambda_{L S P R}=517 \mathrm{~nm}$ (black spectrum). After encapsulation, the spectrum is dominated by scattering from the PNIPAM shell and the LSPR of the gold cores is barely visible (red spectrum). The final core-shell particles with overgrown cores 
show a strong LSPR at $\lambda_{L S P R}=583 \mathrm{~nm}$ dominating the optical response (blue spectrum).
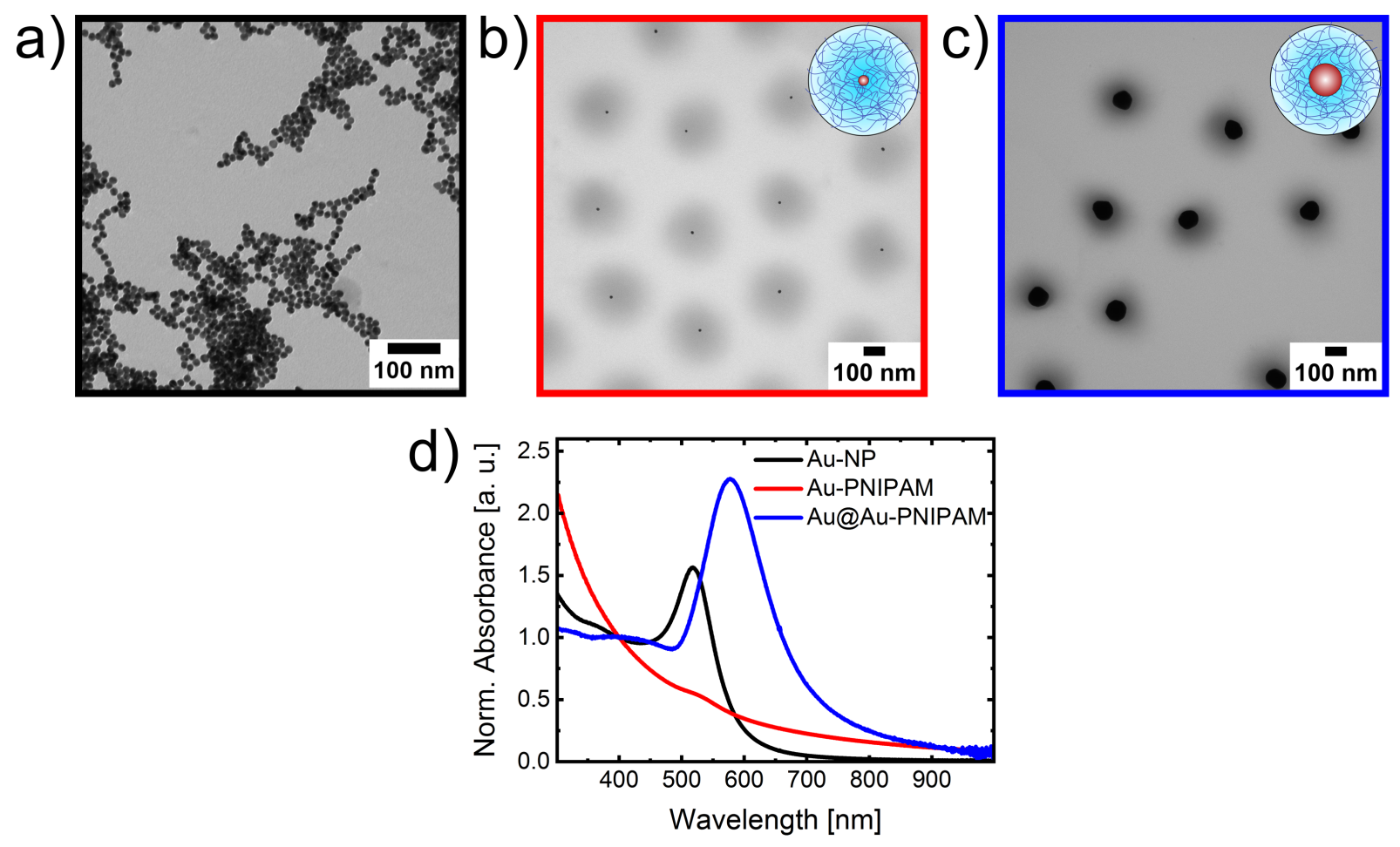

Figure S1: TEM images and UV-Vis spectra of the particles after different steps of preparation. TEM images of the gold cores (a), the core-shell particles after seeded precipitation polymerization (b) and the final core-shell particles after overgrowth of the cores (c). The particles shown in (c) correspond to the particles Au-PNIPAM 367 in the main manuscript. (d) UV-Vis absorbance spectra of the gold cores (black), the Au-PNIPAM particles before (red) and after core growth (blue). All spectra are normalized to the absorbance at $400 \mathrm{~nm}$. 


\section{Microstructure Analysis of Particle Monolayers}

Figure S2 shows images of particle monolayers with $d_{c-c}=458 \mathrm{~nm}$ and $d_{c-c}=567 \mathrm{~nm}$ recorded with a dark-field microscope (DFM) at 100× magnification. The images proof the homogeneity of the samples and the hexagonal order. For both samples the resolution is high enough to resolve single particles in the lattices.
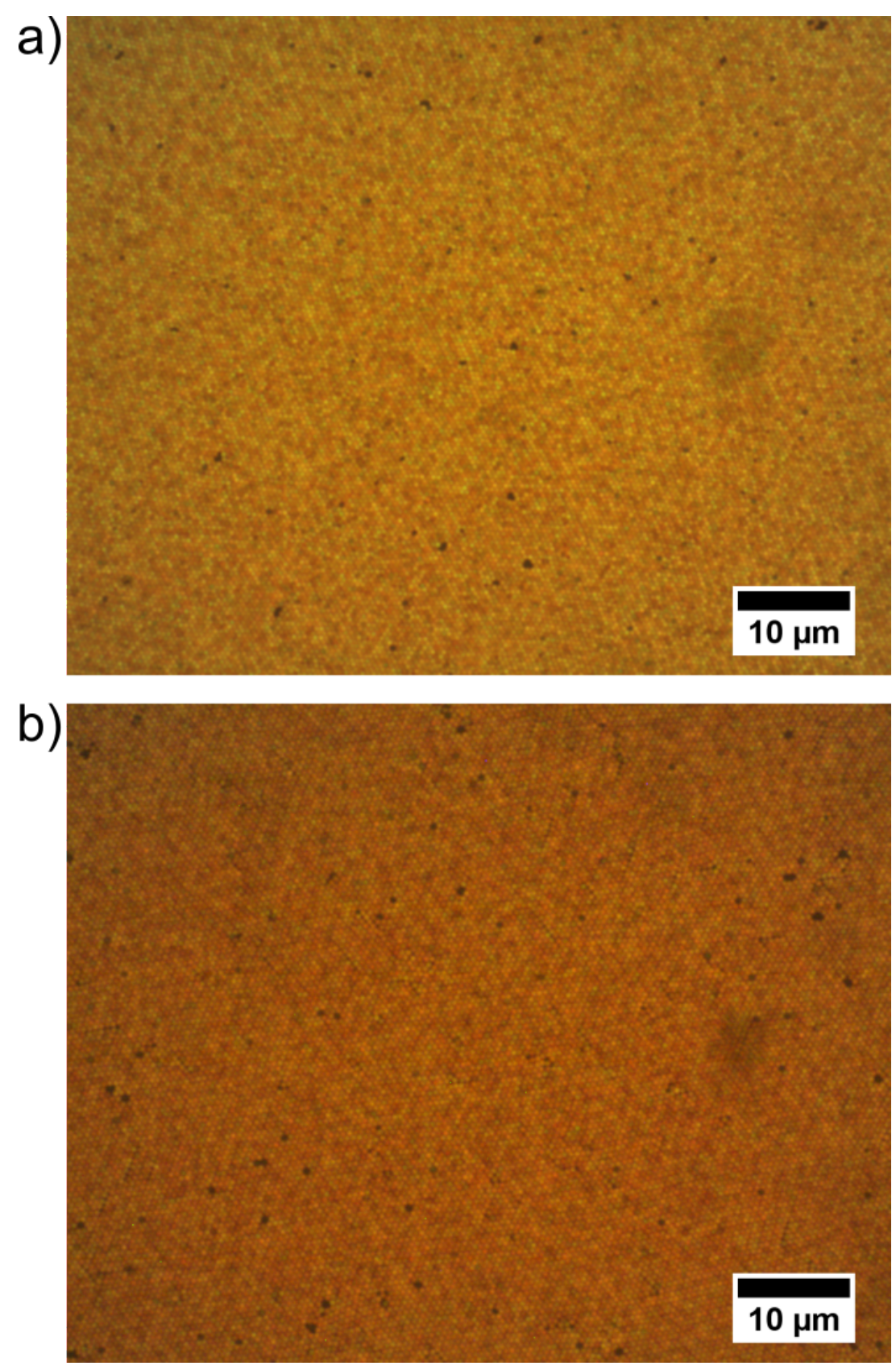

Figure S2: Microstructure analysis by DFM. DFM images recorded at $100 \times$ magnification for a monolayer sample with $d_{c-c}=458 \mathrm{~nm}$ (a) and $d_{c-c}=567 \mathrm{~nm}$ (b). 
Figure S3 (a) shows AFM height profiles of particle monolayers with interparticle distances of $d_{c-c}=378 \mathrm{~nm}, d_{c-c}=448 \mathrm{~nm}$ and $d_{c-c}=573 \mathrm{~nm}$. The insets show fast fourier transformations (FFTs) computed from the AFM images. The FFTs support the hexagonal order of the monolayers through the six-fold symmetry of the arrangement of the Bragg peaks. Several orders of Bragg peaks are visible indicating high local order. Figure S3 (c) shows pair correlation functions $\mathrm{g}(\mathrm{r})$ calculated from the position maps in (b). The red ticks above each $\mathrm{g}(\mathrm{r})$ correspond to the theoretical peak positions for a perfectly ordered hexagonal array with the same interparticle distances as in the experimental monolayers.
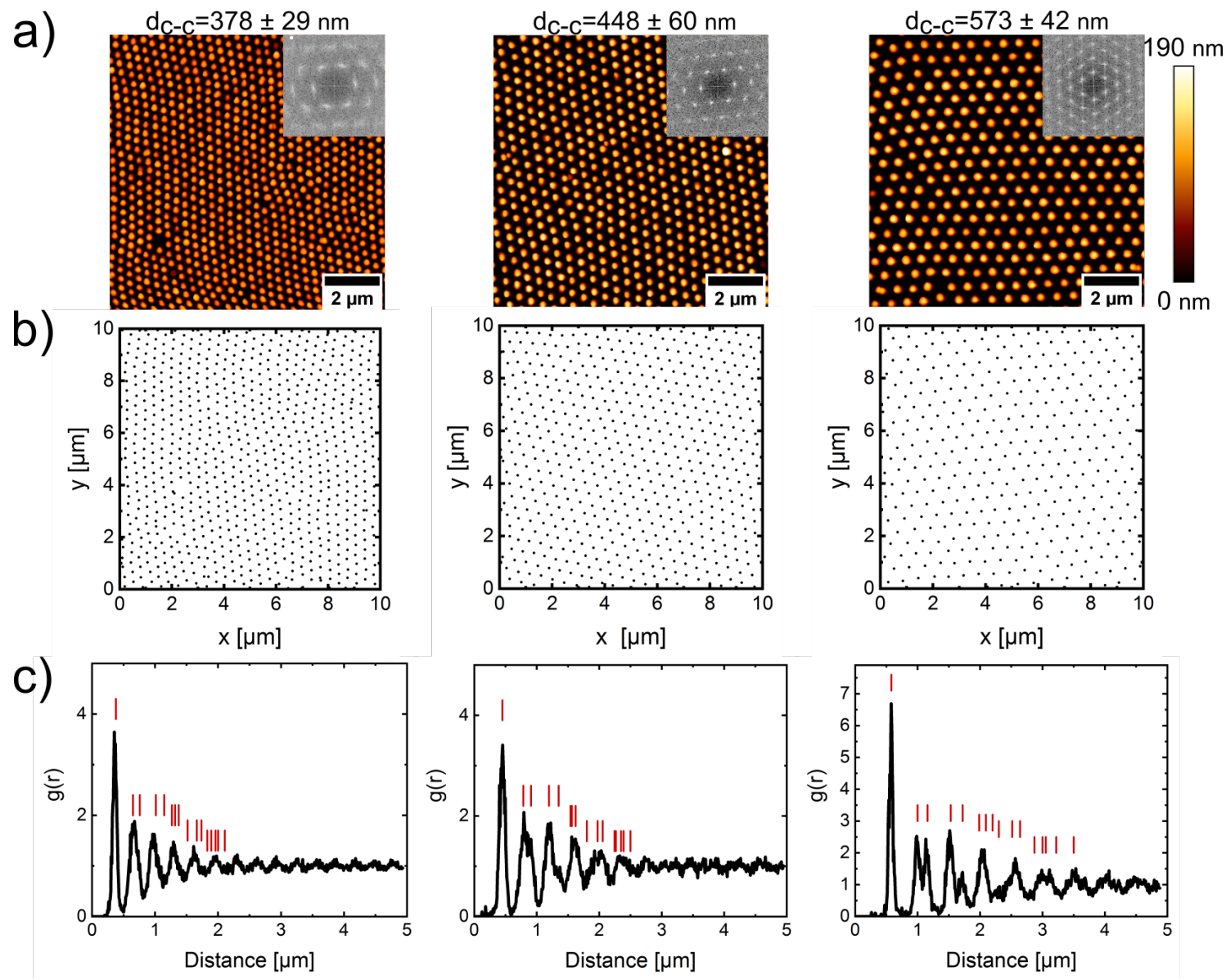

Figure S3: Microstructure analysis by AFM. $10 \times 10 \mu^{2}$ height profile images measured in tapping mode against air (a). The insets show the calculated FFTs. (b) shows the computed position maps of the particle centers in the respective monolayers from (a). (c) pair correlation functions $\mathrm{g}(\mathrm{r})$ of the monolayers. Red ticks indicate the theoretical peak positions for perfectly ordered reference lattices. 
Figure S4 (a) shows AFM height profiles of monolayers with interparticle distances of $d_{c-c}=$ $375 \mathrm{~nm}, d_{c-c}=458 \mathrm{~nm}$ and $d_{c-c}=567 \mathrm{~nm}$ recorded with a scan size of $40 \times 40 \mu \mathrm{m}^{2}$. The size of the single crystalline, hexagonally ordered domains was analyzed adopting the algorithm reported by Hillebrand et al. ${ }^{5}$ Figure $\mathbf{S} 4$ (b) shows the AFM images superimposed with the detected domains in different colors. Each color corresponds to a different domain. Particles marked in white are not associated to any domain. All samples show large crystalline domains. With increasing interparticle distance the monolayers show fewer defects and the domain size increases. Figure S4 (c) shows magnified parts of the monolayer images $(10 \times 10$ $\mu^{2}$ ) with colored triangles connecting three neighboring particles that belong to crystalline domains resulting from our domain analysis. The selected image regions are highlighted by the red squares in (b). 

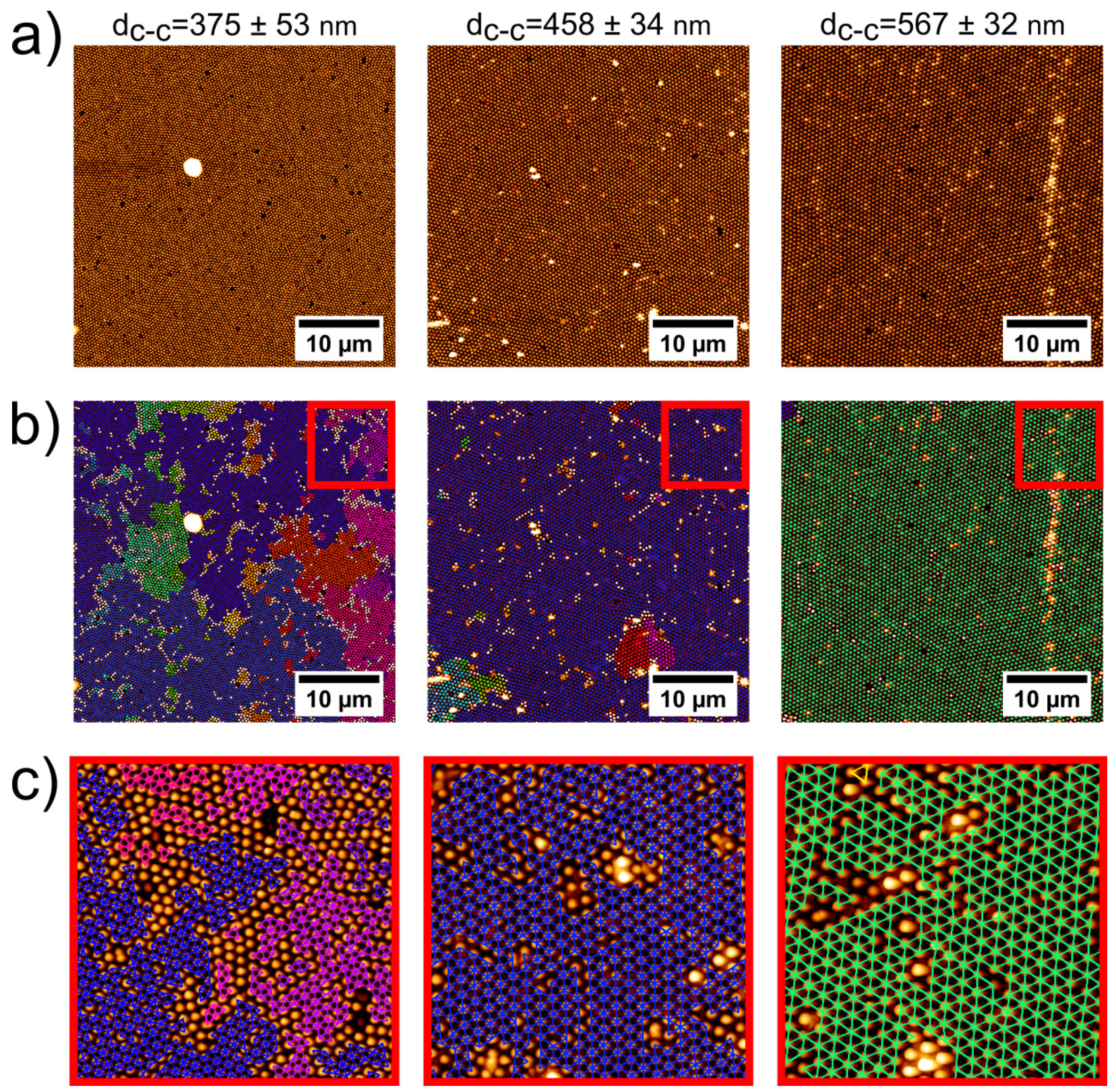

Figure S4: Analysis of domain sizes. $40 \times 40 \mu \mathrm{m}^{2}$ AFM height profile images measured in tapping mode against air for monolayers with $d_{c-c}=375 \mathrm{~nm}, d_{c-c}=458 \mathrm{~nm}$ and $d_{c-c}$ $=567 \mathrm{~nm}$ (a). (b) shows the result of the domain analysis where particles belonging to individual crystalline domains are marked by different colors. Particles marked in white are not associated to any domain. (c) triangulation resulting from domain size analysis. The shown magnified image regions $\left(10 \times 10 \mathrm{\mu m}^{2}\right)$ are highlighted in the corresponding images in (b). 


\section{Experimental Spectra of all Particle Monolayers}

Figure S5 summarizes the absorbance spectra of all particle monolayers for asymmetric (a) and symmetric RI environment (b). The monolayers have values of $d_{c-c}$ in the range of 342 $\mathrm{nm}$ to $573 \mathrm{~nm}$. For the asymmetric RI case all samples show single resonance peaks only. In contrast for the symmetric RI case single resonance peaks are observed for the smaller $d_{c-c}$ while at larger $d_{c-c}$ two resonances appear.
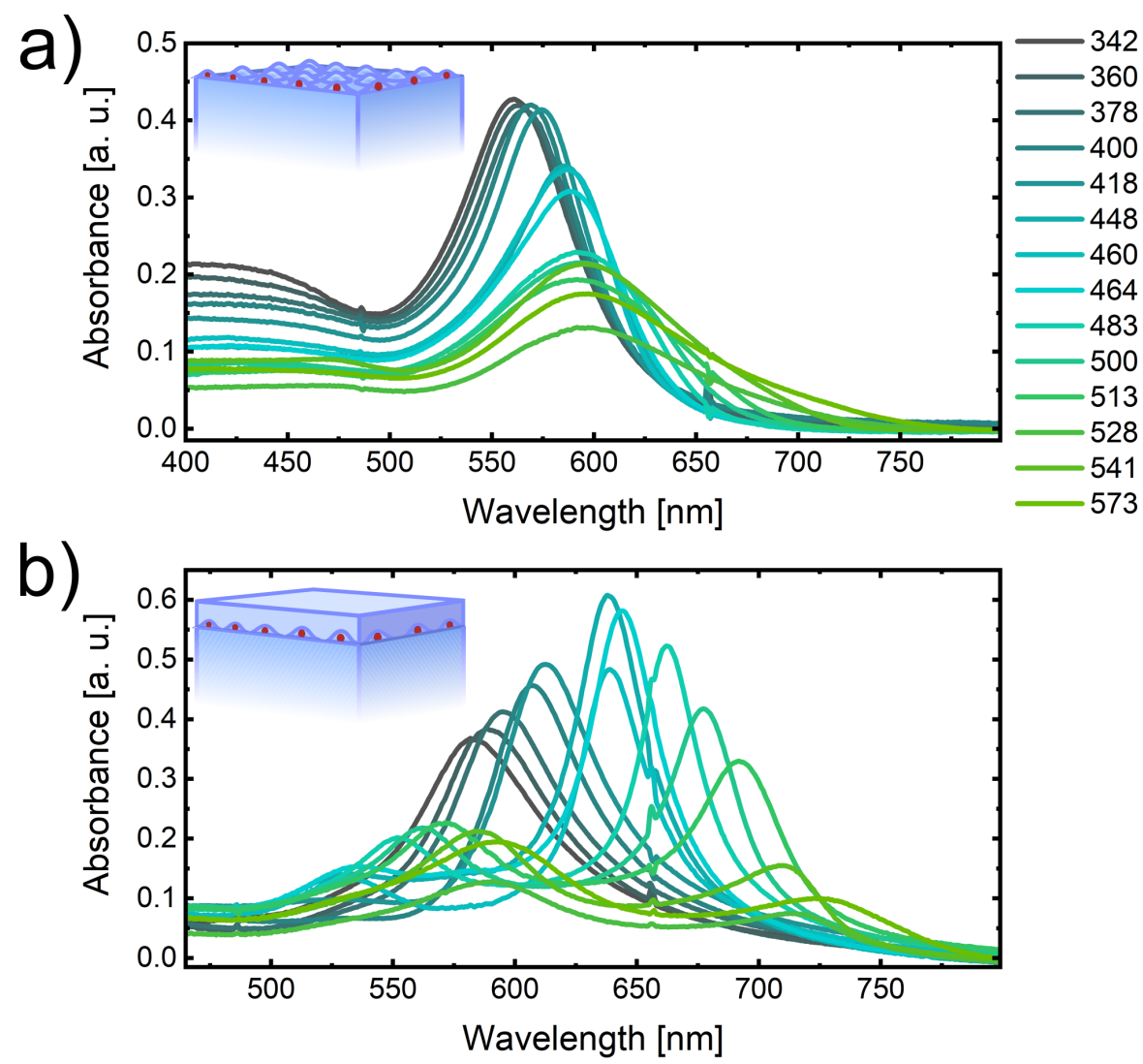

Figure S5: Experimental absorbance spectra for all particle monolayers with different $d_{c-c}$. (a) asymmetric RI environment; (b) symmetric RI environment. The color-coding of the individual spectra is the same in (a) and (b). The insets show schematic depictions of the respective sample geometries with glass as substrate and air as superstrate in (a) and a film of PNIPAM with $300 \mathrm{~nm}$ thickness as superstrate in b).

\section{Optical Response of an Unordered Particle Monolayer}

Figure S6 (a) shows an absorbance spectrum of an unordered Au-PNIPAM ${ }_{336}$ particle monolayer on glass as substrate and air as superstrate. The sample was prepared by spin- 
coating using a highly dilute particle dispersion on a clean glass substrate. The AFM image in (b) shows the respective monolayer with large interparticle distances approximately in the range of 850 to $4000 \mathrm{~nm}$. Due to the large distances and the absence of order, the optical response is comparable to the one of a single particle on glass. The spectrum reveals a single resonance peak at $\lambda_{L S P R}=595 \mathrm{~nm}$.

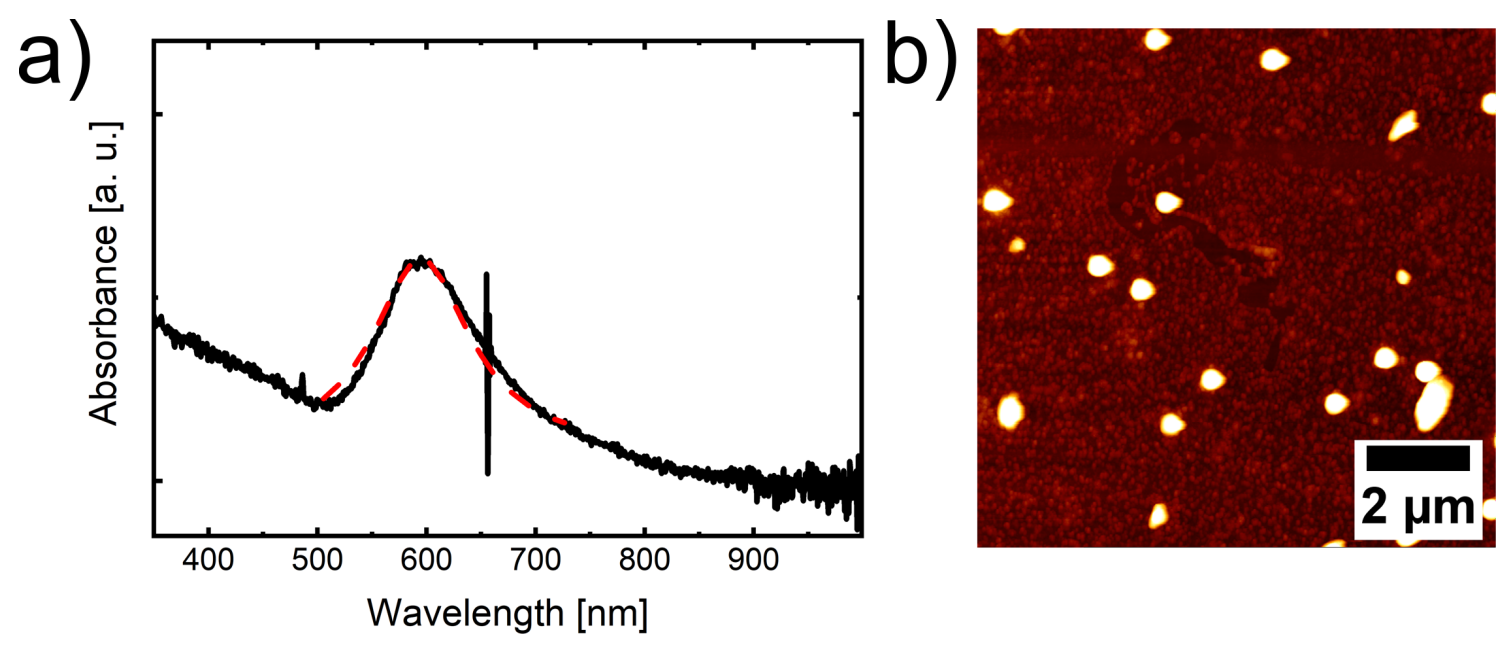

Figure S6: Optical response of an unordered, dilute particle monolayer. (a) Absorbance spectrum of the monolayer. The dashed red line is a Lorentz fit to the LSPR. (b) Corresponding AFM height profile image of the sample $\left(10 \times 10 \mathrm{\mu m}^{2}\right)$.

\section{Local Transmission Spectra of Particle Monolayers}

Figures S7 (a) and (b) show local absorbance spectra of monolayer samples with $d_{c-c}=$ $449 \mathrm{~nm}$ and $d_{c-c}=573 \mathrm{~nm}$. The spectra were recorded in transmission geometry with an optical light microscope using a 50× magnification lens and transmissions were converted to absorbances. For the asymmetric RI case we measure single resonance peaks for both monolayers with resonance positions of $\lambda_{L S P R}=582 \mathrm{~nm}\left(d_{c-c}=449 \mathrm{~nm}\right)$ and $\lambda_{L S P R}=601$ $\mathrm{nm}\left(d_{c-c}=573 \mathrm{~nm}\right)$. In contrast, for symmetric RI both samples feature two resonance peaks where the higher wavelength peaks are assigned to SLRs. The monolayer with $d_{c-c}=$ $449 \mathrm{~nm}$ shows a narrow linewidth SLR at $\lambda_{S L R}=631 \mathrm{~nm}$ and a weak plasmonic contribution at lower wavelengths. The monolayer with $d_{c-c}=573 \mathrm{~nm}$ features a relatively weak SLR at approximately $721 \mathrm{~nm}$ and a significantly more pronounced plasmonic peak at lower 
wavelength. The plasmonic contribution almost resembles the optical response measured for the asymmetric RI case. Overall the spectra are in good agreement to the ones recorded using standard far-field extinction spectroscopy as shown in the main manuscript and in figure S5.
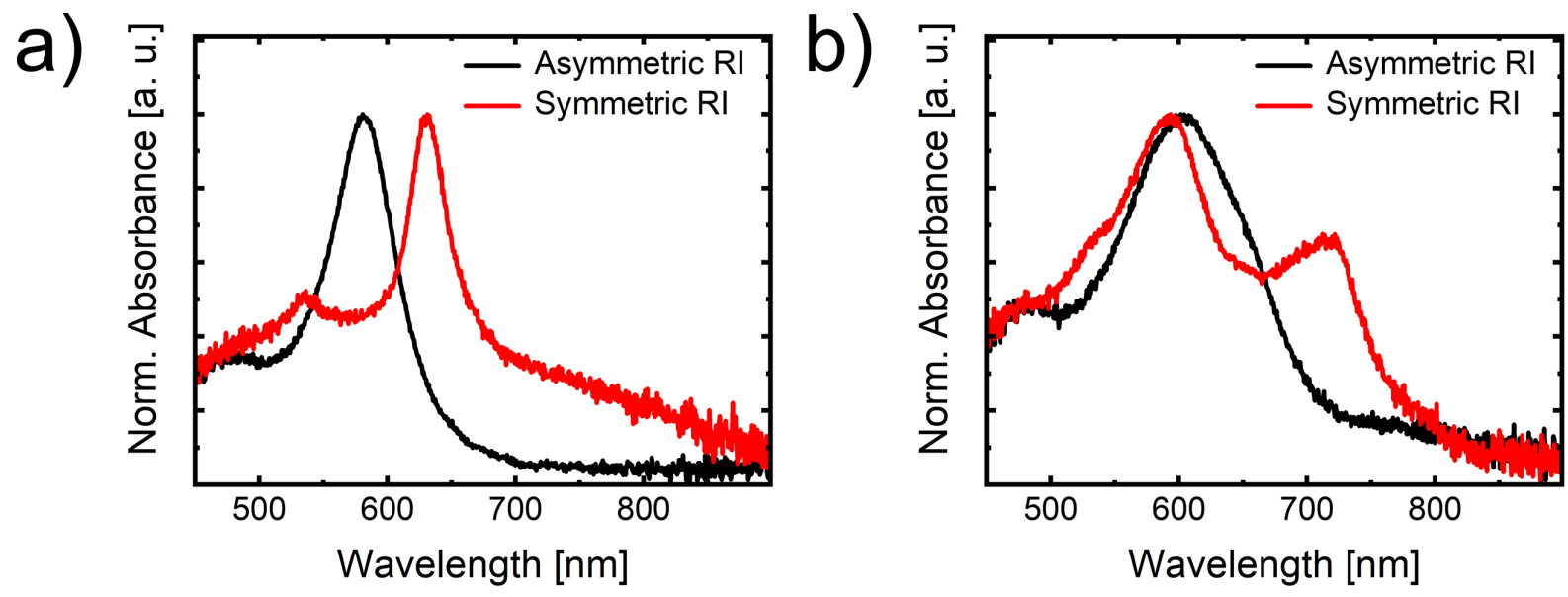

Figure S7: Local optical response. Absorbance spectra of monolayer samples with $d_{c-c}=449$ $\mathrm{nm}$ (a) and $d_{c-c}=573 \mathrm{~nm}$ (b) in asymmetric RI (black lines) and symmetric RI environments (red lines) obtained from transmission measurements with an optical light microscope at $50 \times$ magnification. All spectra were normalized to the maximum peak intensities.

\section{Simulation Layout for FDTD}

Figure S8 shows schematically the measurement geometry from experiment and the simulation layout (FDTD) in the XZ view (infinite lattice). All simulations were performed to match the experimental conditions. Particle sizes as well as distances were used as obtained from the experiments. 


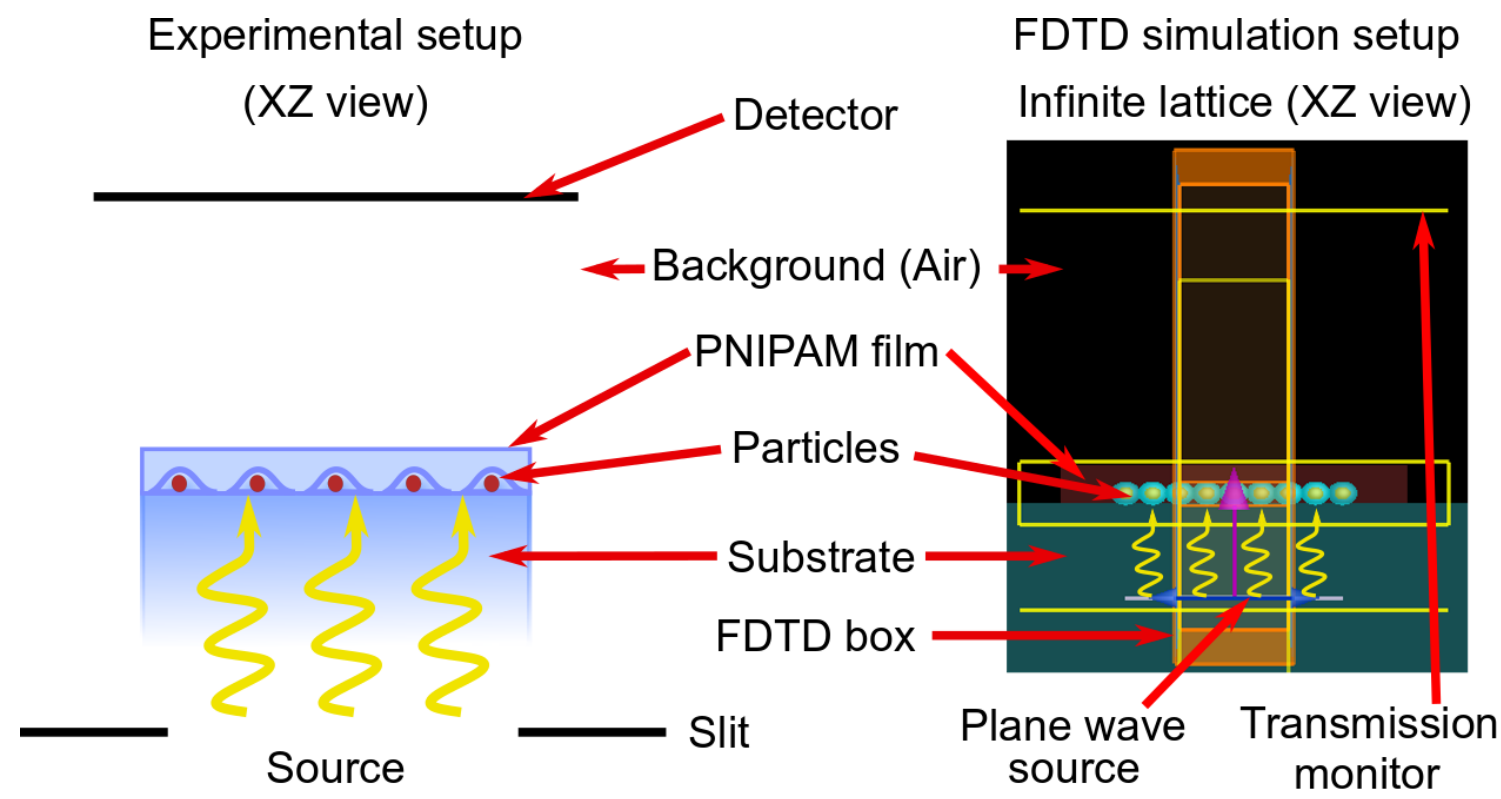

Figure S8: Schematic representation of the experimental transmission measurements and the FDTD simulation conditions for the infinite lattice.

\section{Theoretical Q-factor for Various Domain Sizes}

Figure S9 shows the theoretical Q-factors of the highest intensity resonances obtained from FDTD simulations of particle arrays with different interparticle distances in dependence of the domain size. Compared are results for domain sizes of 2 (19 particles), 3 (37 particles), 4 (61 particles) and 5 (91 particles) as well as for the respective infinite lattices (periodic boundary conditions, PBC). The lattices with $d_{c-c}=400 \mathrm{~nm}$ feature nearly the same Qfactor independent on the domain size. At this interparticle distance diffractive-plasmonic coupling is not supported and thus the resonances are nearly independent on the lattice size. At larger values of $d_{c-c}$ where diffractive-plasmonic coupling results in SLRs, the infinite lattice features significantly higher Q-factors that generally increase with increasing $d_{c-c}$. In contrast the Q-factor of all finite lattices decrease significantly for the largest $d_{c-c}$. 


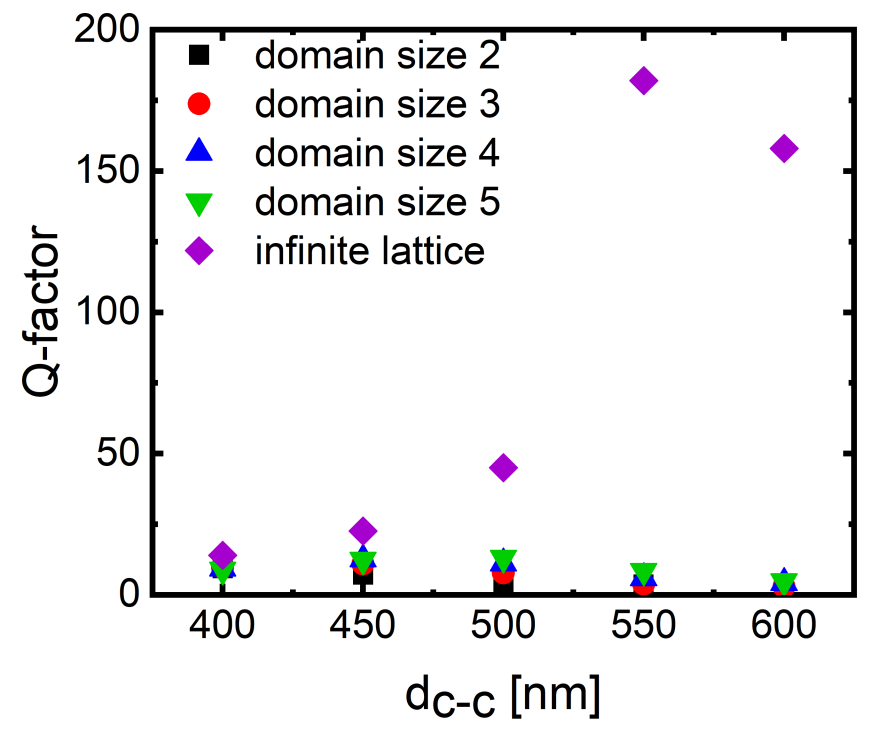

Figure S9: Comparison of Q-factors obtained from FDTD simulations. Compared are the Q-factors of Au nanoparticle arrays with different domain sizes for various $d_{c-c}$ in symmetric $\mathrm{RI}$ environments. The domain size was varied in the range of 2 to 5 . Data are compared to an effectively infinite lattice (PBC).

\section{Theoretical Optical Response in the Strongest Coupling Regime}

Figure S10 shows a calculated extinction spectrum from FDTD simulations of a gold particle lattice with $d_{c-c}=483 \mathrm{~nm}$ in a symmetric RI environment. The simulation was performed with $\mathrm{PBC}$ in $\mathrm{x}$ - and $\mathrm{y}$-direction. The Q-factor of the SLR is 36.9. 


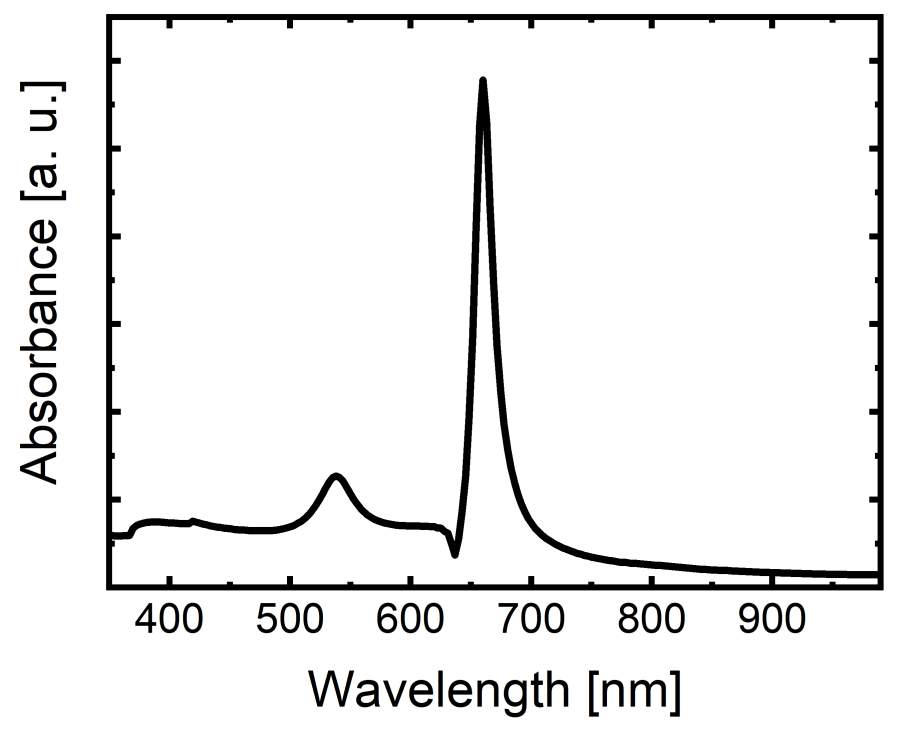

Figure S10: Optical response in the strong coupling regime. Extinction spectrum from FDTD simulation using a gold nanoparticle lattice with $d_{c-c}=483 \mathrm{~nm}$ in a symmetric RI environment and PBC yielding an infinite size lattice.

\section{References}

(1) Turkevich, J.; Stevenson, P. C.; Hillier, J. A study of the nucleation and growth processes in the synthesis of colloidal gold. Discussions of the Faraday Society 1951, 11, 55-75.

(2) Karg, M.; Jaber, S.; Hellweg, T.; Mulvaney, P. Surface Plasmon Spectroscopy of GoldPoly-N-isopropylacrylamide Core-Shell Particles. Langmuir 2011, 27, 820-827.

(3) Rauh, A.; Honold, T.; Karg, M. Seeded precipitation polymerization for the synthesis of gold-hydrogel core-shell particles: the role of surface functionalization and seed concentration. Colloid and Polymer Science 2016, 294, 37-47.

(4) Honold, T.; Volk, K.; Rauh, A.; Fitzgerald, J. P. S.; Karg, M. Tunable plasmonic surfaces via colloid assembly. Journal of Materials Chemistry C 2015, 3, 11449-11457.

(5) Hillebrand, R.; Müller, F.; Schwirn, K.; Lee, W.; Steinhart, M. Quantitative Analysis 
of the Grain Morphology in Self-Assembled Hexagonal Lattices. ACS Nano 2008, 2, 913-920. 